\title{
Arthroscopy and MRI for the knee
}

In its early days arthroscopy was used mainly for diagnosis; it saved many knees from unnecessary arthrotomy. Today, MRI is available to save knees from unnecessary arthroscopy; internal derangements can then be treated by arthroscopic surgery. Arthrotomy for internal derangement is now obsolete and the debate has moved on to the relative roles of arthroscopy and MRI in the management of knee disorders.

MRI is non-invasive, free from known morbidity and is safer and less expensive than arthroscopy. The sensitivity of MRI for meniscal lesions may exceed $90 \%,{ }^{1-3}$ but despite this, diagnostic arthroscopy is often advised too readily. One study showed that $51 \%$ of patients on a waiting-list for arthroscopy for a suspected meniscal lesion were removed after review and MRI. ${ }^{4}$ Should all knees be examined by MRI before arthroscopic surgery?

Sensitivity is not the same as accuracy. Some authors ${ }^{5,6}$ have reported that clinical assessment is more accurate than MRI; others have found no difference. ${ }^{7}$ The accuracy of the clinical diagnosis of meniscal tears is about $75 \%$ to $80 \%$, compared with $88 \%$ to $90 \%$ for MRI. ${ }^{2}$ For lesions of articular cartilage, both the sensitivity and accuracy of MRI are low. ${ }^{8,9}$ Symptomatic meniscal lesions demonstrated by MRI will still need arthroscopic surgery and it can be argued that the MRI was unnecessary.

Many needless arthroscopies will be performed if every MRI report is taken at face value. The clinical problem is to try to avoid MRI for patients who definitely need therapeutic arthroscopy and yet to prevent invasive arthroscopy when there is no surgically treatable lesion. Carmichael et al review the cost-effectiveness of MRI in a paper on p. 624 . They compared the proportions of negative arthroscopies and positive MRIs for a surgeon with a special interest in the knee with those for a group of general orthopaedic surgeons. The specialist knee surgeon performed fewer negative arthroscopies and had more positive MRI scans than his colleagues. This suggests that better clinical skills allowed a more economical use of resources. The study did not show how many patients were managed without either arthroscopy or MRI; a detailed review to demonstrate the value of this clinical judgement would be instructive.

Only an experienced clinician can decide if a lesion demonstrated by MRI is the likely cause of symptoms. Reports by radiologists may present particular difficulties: a bright signal due to myxoid degeneration of the posterior third of the medial meniscus is sometimes described as a 'tear' ${ }^{3}$ or, worse, as a 'persistent tear' if the MRI has followed an arthroscopic meniscectomy. Areas of increased bone density after trauma have been described, for want of a better term, as 'multiple microfractures'. Such reports should be disregarded if they are inconsistent with the clinical findings, but it is clear that medicolegal difficulties, as well as those of management, will arise when the terms used to describe plain radiographs are applied uncritically to MR images.

MRI is a considerable advance and may well preserve patients from unnecessary operations, but it is not infallible and its technology should not dazzle clinicians. A knee which is producing definite mechanical symptoms will need arthroscopic surgery whatever is shown on MRI; the latter is therefore unnecessary. If there are no mechanical symptoms MRI may be indicated to exclude any treatable pathology. Surgeons who are less expert in this specialist field are well advised to request MRI in preference to arthroscopy for a doubtful case, but those who are confident about their management of knee disorders may need neither. MRI is very useful but, as was once said of arthroscopy, it is no substitute for clinical acumen.

D. J. DANDY

\section{REFERENCES}

1. Boeree NR, Watkinson AF, Ackroyd CE, Johnson C. Magnetic resonance imaging of meniscal and cruciate injuries of the knee. J Bone Joint Surg [Br] 1991;73-B:452-7.

2. MacKenzie R, Palmer CR, Lomas DJ, Dixon AK. Magnetic resonance imaging of the knee: diagnostic performance studies. Clin Radiol 1996;51:251-7.

3. Dixon AK. Editorial. Magnetic resonance of the knee. J Bone Joint Surg [Br] 1996;78-B:174-5.

4. Williams RL, Williams LA, Watura R, Fairclough JA. Impact of MRI on a knee arthroscopy waiting list. Ann R Coll Surg Engl 1996; 78:450-2.

5. Rose NE, Gold SM. A comparison of accuracy between clinical examination and magnetic resonance imaging in the diagnosis of meniscal and anterior cruciate ligament tears. Arthroscopy 1996;12: 398-405.

6. Miller GK. A prospective study comparing the accuracy of the clinical diagnosis of meniscus tear with magnetic resonance imaging and its effect on clinical outcome. Arthroscopy 1996;12:406-13.

7. Muellner T, Weinstabl R, Schabus R, Vécsei V, Kainberger F. The diagnosis of meniscal tears in athletes: a comparison of clinical and magnetic resonance imaging investigations. Am J Sports Med 1997; 25:7-12.

8. Spiers ASD, Meagher T, Ostlere SJ, Wilson DJ, Dodd CAF. Can MRI of the knee affect arthroscopic practice? a prospective study of 58 patients. J Bone Joint Surg [Br] 1993;75-B:49-52.

9. Ochi M, Sumen Y, Kanda T, Ikuta Y, Itoh K. The diagnostic value and limitation of magnetic resonance imaging on chondral lesions in the knee joint. Arthroscopy 1994;10:176-83. 